\title{
Development of Anthracnose on Grain Sorghum Hybrids Inoculated with Recently Described Pathotypes of Colletotrichum sublineolum Found in Arkansas
}

\author{
J. W. Moore, Former Senior Graduate Assistant, M. Ditmore, Research Associate, and D. O. TeBeest, University \\ Professor, Department of Plant Pathology, University of Arkansas, Fayetteville 72701
}

\begin{abstract}
Moore, J. W., Ditmore, M., and TeBeest, D. O. 2010. Development of anthracnose on grain sorghum hybrids inoculated with recently described pathotypes of Colletotrichum sublineolum found in Arkansas. Plant Dis. 94:589-595.

Sorghum anthracnose, caused by Colletotrichum sublineolum, is found in most grain-sorghumproducing areas, including Arkansas. Yield losses can be severe in susceptible sorghum hybrids, suggesting that host resistance will continue to be critical for maintaining high yields. However, genetic control is often unsuccessful because of haplotype and pathotype variability within the C. sublineolum population. The objective of this research was to assess the levels of resistance of selected grain sorghum hybrids to isolates of the recently described pathotypes of $C$. sublineolum found in Arkansas. Field experiments were conducted in 2005 and 2006 to evaluate the reaction of 10 sorghum hybrids to eight $C$. sublineolum pathotypes by collecting anthracnose severity ratings at $7,14,21$, and 28 days after inoculation. Based on these disease severity ratings, the area under the disease progress curve was calculated for each pathotype by hybrid interaction. These data showed that different levels of resistance are available in the current grain sorghum hybrids to different pathotypes. Cargill $888 \mathrm{Y}$ was resistant to all pathotypes in both years. However, Asgrow A571, DEKALB DKS53-11, FFR 318, and Pioneer 84G62 were moderately susceptible to susceptible to all pathotypes tested in both years. Pathotype 9 caused the most disease on 7 of the 10 hybrids evaluated in 2005 but, in 2006, pathotypes 12 and 13 surpassed pathotype 9 for disease severity on 9 of the 10 hybrids. More work is needed to assess host resistance and the effect of sorghum anthracnose on yield parameters of grain sorghum hybrids planted in Arkansas.
\end{abstract}

Colletotrichum sublineolum (Henn.) (formerly C. graminicola (Ces.) G.W. Wilson), the causal agent of sorghum anthracnose, is ubiquitous in most of the grain sorghum (Sorghum bicolor)-producing regions of the world. This pathogen can cause severe yield losses in susceptible sorghum hybrids $(10,22)$ by damaging all aerial parts of the plant, including leaves, stalks, peduncles, and panicles $(9,24,25)$. Host plant resistance is the most reliable control strategy for this disease but genetic control has also been unsuccessful because of the high variability within the population of this pathogen $(1,4,17)$. Several pathotypes and haplotypes of this fungus have been reported $(1,4,6,15,18,21,23)$, denoting the variability of this plant pathogen. Grain sorghum has been shown to express dilatory resistance toward $C$. sublineolum but differential interactions between pathogen races or pathotypes and

Corresponding author: D. O. TeBeest

E-mail: dtebeest@uark.edu

Current address of J. W. Moore: Senior Station Superintendent, Cimarron Valley Research Station, Oklahoma State University, Perkins 74059.

Accepted for publication 26 January 2010.

doi:10.1094/PDIS-94-5-0589

(c) 2010 The American Phytopathological Society sorghum genotypes have also been observed $(4-6,14)$. Sorghum anthracnose was first documented in Arkansas in 1985 and has since been economically important to sorghum producers due to reduction in grain yields (6).

Preliminary data from previous experiments conducted in Arkansas have shown that grain sorghum hybrids reacted differently to anthracnose, suggesting that different genes condition resistance (19). Boora et al. (2) reported that sorghum anthracnose resistance is inherited as a single-gene recessive trait when a resistant cultivar (SC326-6) and a susceptible cultivar (BTX 623) were crossed.

Recently, new pathotypes of $C$. sublineolum were isolated and identified in Arkansas (15). The existence of these new pathotypes could also explain the variability noted by TeBeest et al. (19). Because of the variability in the sorghum germplasm available to producers and the existence of these undescribed pathotypes, grain sorghum hybrid recommendations to Arkansas producers have been difficult. Therefore, the objectives of this research were to assess levels of resistance of commonly grown grain sorghum hybrids to endemic C. sublineolum pathotypes.

\section{MATERIALS AND METHODS}

Field experiments. Field experiments were conducted in 2005 and 2006 at the
University of Arkansas Main Experiment Station located at Fayetteville. To reduce background field inoculum interference, experiments were planted in fields that were not historically used for grain sorghum research. For example, the 2005 field trial was planted in an area used for annual ryegrass (Lolium multiflorum Lam.) research and the 2006 experiment was planted in a field used for soybean (Glycine $\max (\mathrm{L}$.$) Merr.) breeding. Experimen-$ tal sites were prepared by disking and then each experiment was planted on raised beds. Herbicides, including atrazine (AAtrex 4L; Syngenta Crop Protection, Greensboro, NC) at $1.12 \mathrm{~kg}$ a.i./ha plus metolachlor (Dual Magnum; Syngenta Crop Protection) at $1.12 \mathrm{~kg}$ a.i./ha, were applied preemergence to the entire field each year as a general weed control maintenance application. Additional hand weeding was performed when necessary. Furrow irrigation was applied as needed to help simulate cultural practices that are commonly used where grain sorghum is grown commercially within the state.

Collection, isolation, and storage of $\boldsymbol{C}$. sublineolum. C. sublineolum isolates used in this study were isolated from grain sorghum grown in Arkansas and pathotyped on sorghum breeding lines as previously described by Moore et al. (15). All singlespore cultures were stored in a cryofreezer $\left(-80^{\circ} \mathrm{C}\right)$ located in the Department of Plant Pathology at the University of Arkansas in Fayetteville. The eight isolates used in this study included the following: AR595 (pathotype 7), AR577 (pathotype 8), AR571 (pathotype 9), AR612 (pathotype 10), AR581 (pathotype 11), AR569 (pathotype 12), AR579 (pathotype 13), and AR555 (pathotype 14). Additional information for each isolate can be found in Table 1.

Inoculum preparation and inoculation of field plots. The isolates described above were grown on Emerson's yeast extract agar (YpSS) medium (4 $\mathrm{g}$ of yeast extract, $15 \mathrm{~g}$ of soluble starch, $1 \mathrm{~g}$ of $\mathrm{K}_{2} \mathrm{HPO}_{4}, 0.5 \mathrm{~g}$ of $\mathrm{MgSO}_{4}$, and $16 \mathrm{~g}$ of agar per liter), as described previously by $\mathrm{Li}$ (11), and placed in an incubator at $25^{\circ} \mathrm{C}$ under fluorescent lights (General Electric warm white brightstik 33, 725 lumens) with a 16-h photoperiod to promote sporulation. All eight isolates, each representing a single pathotype, were used in the 2005 and 2006 field experiments. Inoculum was prepared by flooding 10-day-old cultures 
with $20 \mathrm{ml}$ of distilled water and gently scraping the surfaces of the cultures with a sterile loop to dislodge the conidia. Then, each conidial suspension was filtered through three layers of cheesecloth and adjusted to a final spore concentration of approximately $1 \times 10^{6}$ spores $/ \mathrm{ml}$ using a hemacytometer. Inoculum was applied in the field to all sorghum hybrid plants within a plot at a rate of $25 \mathrm{ml} /$ plot using a Solo Hand Spritzer single nozzle pump-up sprayer on 12 August 2005 (72 days after planting; early flowering stage) and $4 \mathrm{Au}-$ gust 2006 (71 days after planting; early flowering stage). The sprayer was not calibrated to deliver a certain amount of inoculum per unit of time. Instead the single, adjustable nozzle was adjusted to produce a fine-droplet-size spray pattern so that the inoculum (25 ml/plot) was distributed evenly over the upper leaves of all plants in each plot.

Host reactions. Sorghum entries were evaluated for reaction to each of the pathotypes as previously described (15). All plants in each plot were rated and the lesion type representing the majority (or consensus) of the lesions on all plants per plot was reported as the lesion type reaction for that host-pathotype combination. Ratings were based on lesions observed 28 days after inoculation (DAI) using the following scale: resistant $(\mathrm{R})=$ very small lesions $(<1 \mathrm{~mm})$, with no evidence of lesion expansion or increase in size; moderately resistant $(\mathrm{MR})=$ lesions larger $(1$ to $3 \mathrm{~mm}$ ) but not generally associated with sporulation or significant chlorosis; moderately susceptible $(\mathrm{MS})=$ lesions larger $(4$ to $6 \mathrm{~mm}$ ) and associated with chlorosis and some sign of sporulation on a few lesions; and susceptible $(\mathrm{S})=$ lesions generally large $(>6 \mathrm{~mm})$, with significant chlorosis and sporulation occurs on $>50 \%$ of the lesions.

Anthracnose severity. In addition to the lesion type reaction ratings, disease severity was determined for each plot at 7, 14, 21 , and 28 DAI using a modified HorsfallBarratt scale, where $0=$ none, $1=0$ to 3,2 $=4$ to $6,3=7$ to $12,4=13$ to $25,5=26$ to $50,6=51$ to $75,7=76$ to $88,8=89$ to $94,9=95$ to 97 , and $10=98$ to $100 \%$. Ratings were converted to the midpoint values before analysis.

After the 28-DAI ratings, five lesions were collected at random from each main plot to confirm pathotype identity using amplified fragment length polymorphism (AFLP). Details of the AFLP procedure are reported in previous work (14). Briefly, the pathogen was isolated from the leaf tissue and placed on YpSS agar medium in an incubator at $25^{\circ} \mathrm{C}$ under fluorescent lights as described above to promote colony growth. DNA was then extracted, digested, and amplified using EcoRI selective primers $(E c o R I+A G$ and $+\mathrm{GC})$ that were labeled at the $5^{\prime}$ end with 6 Fam fluorescent dye (Sigma Genosys, Houston). Each sample was loaded into an Applied Biosystems Genetic Analyzer (model 3100; Foster City, CA) that produced AFLP profiles which were assessed individually. Peaks from these profiles were scored for the presence $(=1)$ or absence $(=0)$ of bands using GeneMapper 4.0 (Applied Biosystems), producing a DNA profile for each

Table 1. Location, grain sorghum hybrid from which isolates were collected, and pathotype designation for the Colletotrichum sublineolum isolates used in the 2005 and 2006 experiments

\begin{tabular}{lllc}
\hline Isolate & \multicolumn{1}{c}{ Location $^{\mathbf{a}}$} & \multicolumn{1}{c}{ Hybrid } & Pathotype $^{\mathbf{b}}$ \\
\hline AR595 & Pine Tree, AR & Triumph TR461 & 7 \\
AR577 & Marianna, AR & Monsanto X234 & 8 \\
AR571 & Marianna, AR & DEKALB DKS54-00 & 9 \\
AR612 & Marianna, AR & Dyna Gro X17F30 & 10 \\
AR581 & Marianna, AR & Monsanto X234 & 11 \\
AR569 & Marianna, AR & Terral TV97H17 & 12 \\
AR579 & Marianna, AR & Monsanto X234 & 13 \\
AR555 & Forrest City, AR & Asgrow A571 & 14 \\
\hline
\end{tabular}

${ }^{\text {a }}$ City closest to the field where isolates were collected.

${ }^{\mathrm{b}}$ Isolates were classified as a certain pathotype based on their virulence to eight host differentials (15).

Table 2. Analysis of variance $P$ values for the effects of isolates and grain sorghum hybrids on disease severity and area under the disease progress curve (AUDPC) over 2 years ${ }^{\mathrm{a}}$

\begin{tabular}{lrccccc}
\hline Source of variation & df & 7 DAI (\%) & 14 DAI (\%) & 21 DAI (\%) & 28 DAI (\%) & AUDPC \\
\hline Isolate & 7 & 0.0418 & 0.0001 & 0.0001 & 0.0001 & 0.0001 \\
Hybrid & 9 & 0.0001 & 0.0001 & 0.0001 & 0.0001 & 0.0001 \\
Isolate-hybrid & 63 & 0.0011 & 0.0001 & 0.0001 & 0.0001 & 0.0001 \\
Year & 1 & 0.0001 & 0.0001 & 0.0001 & 0.0001 & 0.0001 \\
Isolate-year & 7 & 0.0004 & 0.0001 & 0.0001 & 0.0001 & 0.0001 \\
Hybrid-year & 9 & 0.0001 & 0.0001 & 0.0090 & 0.0001 & 0.0001 \\
Isolate-hybrid-year & 63 & 0.1329 & 0.0001 & 0.0012 & 0.0001 & 0.0001 \\
\hline
\end{tabular}

${ }^{a}$ Isolates AR595, AR577, AR571, AR612, AR581, AR569, AR579, and AR555 represent pathotypes 7, 8, 9, 10, 11, 12, 13, and 14, respectively. Hybrids = Asgrow A571, BTX 623, Cargill 888Y, DEKALB DKS53-11, DEKALB DKS54-00, Dyna Gro 780B, FFR 318, Pioneer 84G62, Terral TV1050, and Terral TV96H81. Disease severity ratings were collected at 7, 14, 21, and 28 days after inoculation (DAI) for 2005 and 2006. pathotype. To confirm pathotype identity, each sample was then compared with the original AFLP profile that was generated for each pathotype prior to initiating the experiments (data not shown).

The area under the disease progress curve (AUDPC) was calculated for each pathotype and grain sorghum hybrid combination over the four rating dates within each year using the following equation:

$A U D P C=\sum_{i}^{n-1}\left(\frac{y_{i}+y_{i+1}}{2}\right)\left(t_{i+1}-t_{i}\right)$

in which $t_{i}$ is the time at $i$ th observation, $t_{i+1}$ is the time at $(i+1)$ th observation, $y_{\mathrm{i}}$ is the disease severity (\%) at $i$ th observation, and $y_{i+1}$ is the disease severity at $(i+1)$ th observation (3).

Experimental design and data analysis. Experiments were designed each year as a split plot with pathotypes as the main plot and grain sorghum hybrids as the split plot with four replications. The main plots consisted of eight different $C$. sublineolum isolates representing eight different pathotypes and deionized water only (nontreated control) for a total of nine main plots. Split plots consisted of 10 grain sorghum hybrids, including Asgrow A571, BTX 623, Cargill 888Y, DEKALB DKS 53-11, DEKALB DKS 54-00, Dyna Gro 780B, FFR 318, Pioneer 84G62, Terral TV1050, and Terral TV96H81. Grain sorghum hybrids within each main plot were planted in single-row plots, $2 \mathrm{~m}$ long and approximately $1 \mathrm{~m}$ apart, on 1 June 2005 and 25 May 2006. Buffer rows of corn extending $2 \mathrm{~m}$ long were planted between each main plot to reduce cross contamination between main plots (isolates).

Disease severity ratings and AUDPC data were analyzed using the JMP 8 statistical software package (SAS Institute, Cary, NC). Analysis of each disease severity rating and the AUDPC were conducted as a split-split plot with $C$. sublineolum pathotypes as the main parameter, grain sorghum hybrids as the split parameter, and year as the split-split parameter. Least significant differences $(P=0.05)$ were calculated for the significant main effects and interaction means using the guidelines provided by Little and Hills (13). Data presented are the least square means for disease severity ratings and AUDPCs for four replications for each year.

\section{RESULTS}

Results showed that there were significant isolate-sorghum hybrid interactions for the 7 DAI rating and significant isolate-sorghum hybrid-year interactions for the 14-, 21-, and 28-DAI ratings (Table 2). Similarly, there were significant interactions for isolate-sorghum hybrid-year for AUDPC. This suggests that the interactions between the environment and the pathosystem were different in each year and may reflect the different environ- 
mental conditions in 2005 compared with 2006. In 2005, approximately $38.1 \mathrm{~mm}$ of rainfall occurred between the time of inoculation and the last rating date (28 DAI). In contrast, approximately $286.8 \mathrm{~mm}$ of precipitation occurred in 2006. Anthracnose was not found on any of the nontreated check plots, which supported the assumption that background inoculum should not be a problem in these fields chosen for our experiments because these fields were not used for grain sorghum research. The lack of disease on the nontreated check plots also confirmed that the corn buffer rows were successful in preventing cross contamination between main plots (pathotypes).

Based on the lesion type reactions at 28 DAI, Cargill 888 Y was resistant to all isolates evaluated (Table 3). However, BTX 623 and DEKALB DKS54-00 mainly produced moderately resistant reactions to most of the isolates. In 2006, moderate reactions occurred on 6 of the 10 sorghum hybrids evaluated for isolates AR612 and AR581 whereas 7 hybrids exhibited a moderate reaction to isolate AR555 in 2005 (Table 3). Lesion type reactions were similar between years for most of the sorghum hybridisolate interactions.

Isolates AR577 and AR571 both caused significantly lower levels of disease in
2006 than in 2005 on 6 of the 10 sorghum hybrids according to the AUDPC (Table 4). These differences may be attributed to the different environmental conditions for each year which may have caused a delay in the disease progress in 2006 compared with 2005. Of the 10 sorghum hybrids evaluated, only 2 hybrids (Cargill $888 \mathrm{Y}$ and DEKALB DKS54-00) did not show significant differences in AUDPC between years for the same isolate. Sorghum hybrid FFR 318 had significantly higher AUDPC values in 2006 than in 2005 for isolates AR581, AR569, AR579, and AR555. In addition, AR555 produced significantly more disease on FFR 318, Pioneer 84G62, and Terral TV1050 in 2006 than in 2005 (Table 4).

Anthracnose severity was similar on Asgrow A571 for all isolates at 7 DAI in 2005 (Fig. 1A). By 14 DAI, isolates AR595, AR577, and AR571 caused significantly more disease, reaching approximately $18 \%$ disease severity on Asgrow A571. In contrast, isolates AR612, AR581, and AR555 produced significantly lower levels of disease on this hybrid at 14 DAI. Severity significantly increased on Asgrow A571 by 21 DAI for isolates AR571 (pathotype 9) and AR581 (pathotype 11) and, by 28 DAI, these two isolates produced the highest amount of disease on
Asgrow A571 in 2005. In 2006, all isolates caused similar amounts of disease on Asgrow A571 when compared with 2005 for 7 and 14 DAI (Fig. 2A). However, at 21 DAI in 2006, disease severity was significantly higher for isolate AR569 (pathotype 12). This isolate produced approximately $10 \%$ more disease than the two isolates (AR571 and AR581) that caused the highest levels of disease in 2005 by 21 DAI. By 28 DAI, isolate AR569 had caused approximately $30 \%$ more disease than all of the other isolates on Asgrow A571 (Fig. 2A).

Disease severity on BTX623 in 2005 was lower for all isolates and for all rating dates when compared with the amount of disease on Asgrow A571 (Fig. 1B). Isolates AR612 and AR581 produced severity ratings of approximately $19 \%$ while the remaining isolates had severity ratings around $10 \%$. In 2006, severity ratings on BTX 623 were below $10 \%$ for all ratings dates, except for isolate AR612, which produced approximately 15 and $25 \%$ disease at 21 and 28 DAI, respectively (Fig. 2B).

Cargill $888 \mathrm{Y}$ was resistant to all isolates evaluated in both years. The severity ratings were slightly above $0 \%$ at all rating dates in both years, which indicate that each isolate was capable of infecting Cargill $888 \mathrm{Y}$ but was unable to colonize and

Table 3. Reactions of 10 grain sorghum hybrids to eight different Colletotrichum sublineolum isolates for 2005 and 2006

\begin{tabular}{|c|c|c|c|c|c|c|c|c|c|c|c|c|c|c|c|c|}
\hline \multirow[b]{3}{*}{ Sorghum hybrid } & \multicolumn{16}{|c|}{ Lesion type reaction for $C$. sublineolum isolates ${ }^{a}$} \\
\hline & \multicolumn{2}{|c|}{ AR595 } & \multicolumn{2}{|c|}{ AR577 } & \multicolumn{2}{|c|}{ AR571 } & \multicolumn{2}{|c|}{ AR612 } & \multicolumn{2}{|c|}{ AR581 } & \multicolumn{2}{|c|}{ AR569 } & \multicolumn{2}{|c|}{ AR579 } & \multicolumn{2}{|c|}{ AR555 } \\
\hline & 2005 & 2006 & 2005 & 2006 & 2005 & 2006 & 2005 & 2006 & 2005 & 2006 & 2005 & 2006 & 2005 & 2006 & 2005 & 2006 \\
\hline Asgrow A571 & $\mathrm{S}$ & $\mathrm{S}$ & $\mathrm{S}$ & $\mathrm{S}$ & $\mathrm{S}$ & $\mathrm{S}$ & $\mathrm{S}$ & MS & $\mathrm{S}$ & $\mathrm{S}$ & $\mathrm{S}$ & $\mathrm{S}$ & $\mathrm{S}$ & $\mathrm{S}$ & $\mathrm{S}$ & $\mathrm{S}$ \\
\hline BTX 623 & MS & MR & MS & MR & MS & MR & $\mathrm{S}$ & $\mathrm{S}$ & $\mathrm{S}$ & MS & MS & MR & MS & MR & MS & MS \\
\hline Cargill 888Y & $\mathrm{R}$ & $\mathrm{R}$ & $\mathrm{R}$ & $\mathrm{R}$ & $\mathrm{R}$ & $\mathrm{R}$ & $\mathrm{R}$ & $\mathrm{R}$ & $\mathrm{R}$ & $\mathrm{R}$ & $\mathrm{R}$ & $\mathrm{R}$ & $\mathrm{R}$ & $\mathrm{R}$ & $\mathrm{R}$ & $\mathrm{R}$ \\
\hline DEKALB DKS53-11 & $\mathrm{S}$ & MS & $\mathrm{S}$ & $\mathrm{S}$ & $\mathrm{S}$ & $\mathrm{S}$ & $\mathrm{S}$ & MS & $\mathrm{S}$ & MS & $\mathrm{S}$ & $\mathrm{S}$ & $\mathrm{S}$ & $\mathrm{S}$ & MS & MS \\
\hline DEKALB DKS54-00 & $\mathrm{S}$ & MS & MS & MR & $\mathrm{S}$ & MS & MS & MR & $\mathrm{S}$ & MS & $\mathrm{S}$ & MS & $\mathrm{S}$ & MS & MS & MS \\
\hline Dyna Gro $780 \mathrm{~B}$ & $\mathrm{~S}$ & $\mathrm{~S}$ & $\mathrm{~S}$ & $\mathrm{~S}$ & $\mathrm{~S}$ & $\mathrm{~S}$ & $\mathrm{~S}$ & MS & MS & MR & $\mathrm{S}$ & $\mathrm{S}$ & $\mathrm{S}$ & $\mathrm{S}$ & MR & MR \\
\hline FFR 318 & $\mathrm{~S}$ & $\mathrm{~S}$ & $\mathrm{~S}$ & $\mathrm{~S}$ & $\mathrm{~S}$ & $\mathrm{~S}$ & $\mathrm{~S}$ & $\mathrm{~S}$ & $\mathrm{~S}$ & $\mathrm{~S}$ & $\mathrm{~S}$ & $\mathrm{~S}$ & $\mathrm{~S}$ & $\mathrm{~S}$ & $\mathrm{~S}$ & $\mathrm{~S}$ \\
\hline Pioneer 84G62 & $\mathrm{S}$ & $\mathrm{S}$ & $\mathrm{S}$ & $\mathrm{S}$ & $\mathrm{S}$ & $\mathrm{S}$ & $\mathrm{S}$ & MS & $\mathrm{S}$ & $\mathrm{S}$ & $\mathrm{S}$ & $\mathrm{S}$ & $\mathrm{S}$ & $\mathrm{S}$ & MS & $\mathrm{S}$ \\
\hline Terral TV1050 & $\mathrm{S}$ & $\mathrm{S}$ & $\mathrm{S}$ & $\mathrm{S}$ & $\mathrm{S}$ & $\mathrm{S}$ & MS & MR & MS & MS & $\mathrm{S}$ & MS & $\mathrm{S}$ & MS & MS & MS \\
\hline Terral TV96H81 & $\mathrm{S}$ & $\mathrm{S}$ & $\mathrm{S}$ & $\mathrm{S}$ & $\mathrm{S}$ & $\mathrm{S}$ & $\mathrm{S}$ & S & $\mathrm{S}$ & MR & $\mathrm{S}$ & $\mathrm{S}$ & $\mathrm{S}$ & $\mathrm{S}$ & MS & MS \\
\hline
\end{tabular}

${ }^{\mathrm{a}} \mathrm{R}=$ resistant, $\mathrm{MR}=$ moderately resistant, $\mathrm{MS}$ = moderately susceptible, and $\mathrm{S}=$ susceptible. Lesion type reactions represent the consensus reactions from four replications at 28 days after inoculation.

Table 4. Effect of eight Colletotrichum sublineolum isolates on area under the disease progress curve (AUDPC) for 10 grain sorghum hybrids over 2 years ${ }^{\mathrm{a}}$

\begin{tabular}{|c|c|c|c|c|c|c|c|c|c|c|c|c|c|c|c|c|}
\hline \multirow[b]{3}{*}{ Sorghum hybrid ${ }^{b}$} & \multicolumn{16}{|c|}{ AUDPC for $C$. sublineolum isolates } \\
\hline & \multicolumn{2}{|c|}{ AR595 } & \multicolumn{2}{|c|}{ AR577 } & \multicolumn{2}{|c|}{ AR571 } & \multicolumn{2}{|c|}{ AR612 } & \multicolumn{2}{|c|}{ AR581 } & \multicolumn{2}{|c|}{ AR569 } & \multicolumn{2}{|c|}{ AR579 } & \multicolumn{2}{|c|}{ AR555 } \\
\hline & 2005 & 2006 & 2005 & 2006 & 2005 & 2006 & 2005 & 2006 & 2005 & 2006 & 2005 & 2006 & 2005 & 2006 & 2005 & 2006 \\
\hline Asgrow A571 & 340 & 244 & 323 & 174 & 483 & 265 & 215 & 105 & 365 & 248 & 289 & 464 & 339 & 319 & 105 & 165 \\
\hline BTX 623 & 185 & 37 & 141 & 81 & 221 & 79 & 279 & 268 & 231 & 68 & 123 & 68 & 155 & 50 & 160 & 79 \\
\hline Cargill 888Y & 37 & 37 & 37 & 37 & 37 & 37 & 37 & 37 & 37 & 37 & 37 & 37 & 37 & 37 & 37 & 37 \\
\hline DEKALB DKS53-11 & 142 & 162 & 178 & 124 & 249 & 186 & 134 & 146 & 207 & 108 & 218 & 219 & 200 & 170 & 37 & 89 \\
\hline DEKALB DKS54-00 & 121 & 72 & 126 & 58 & 159 & 103 & 66 & 71 & 140 & 85 & 96 & 101 & 105 & 112 & 37 & 55 \\
\hline Dyna Gro 780B & 316 & 236 & 273 & 155 & 338 & 231 & 162 & 137 & 88 & 53 & 262 & 313 & 293 & 348 & 53 & 60 \\
\hline FFR 318 & 417 & 378 & 455 & 179 & 430 & 382 & 249 & 285 & 383 & 500 & 306 & 514 & 275 & 445 & 121 & 269 \\
\hline Pioneer 84G62 & 296 & 186 & 329 & 166 & 503 & 252 & 238 & 194 & 288 & 303 & 329 & 334 & 349 & 404 & 110 & 228 \\
\hline Terral TV1050 & 210 & 195 & 224 & 125 & 267 & 170 & 87 & 89 & 167 & 127 & 226 & 227 & 167 & 186 & 58 & 150 \\
\hline Terral TV96H81 & 342 & 311 & 467 & 225 & 472 & 319 & 233 & 326 & 112 & 53 & 480 & 467 & 433 & 437 & 80 & 81 \\
\hline
\end{tabular}

a AUDPC was determined based on disease severity ratings collected at 7, 14, 21, and 28 days after inoculation. The values are the means of four replications.

${ }^{\mathrm{b}}$ Least significant difference $(0.05)=80$ to compare grain sorghum hybrids within the same isolate and the same or different year and $=86$ to compare isolates for the same or different grain sorghum hybrid and year. 

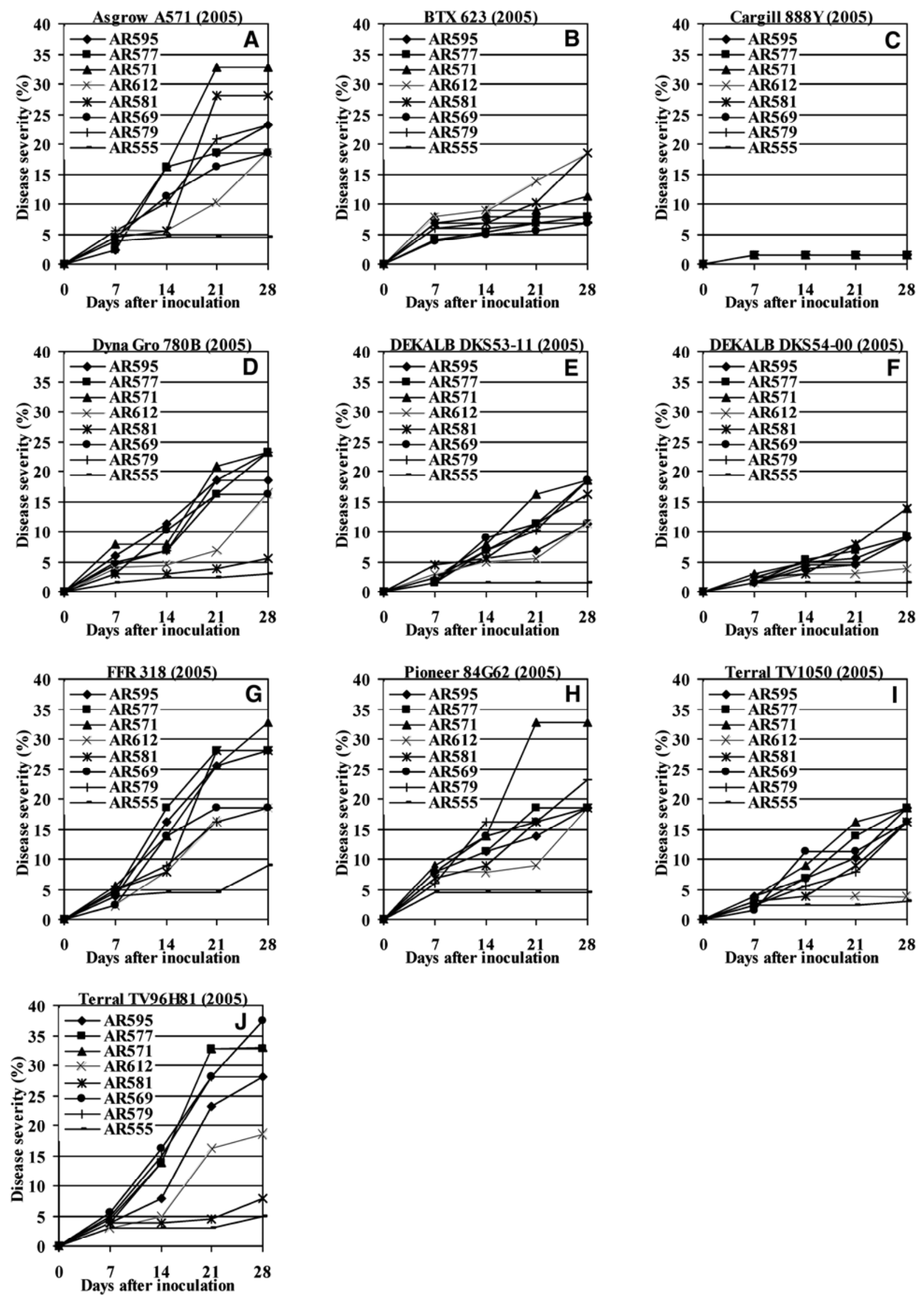

Fig. 1. Anthracnose development on 10 grain sorghum hybrids for eight Colletotrichum sublineolum isolates in the 2005 field test. Data points represent the average of four replications. Least significant differences that compare isolates for the same or different grain sorghum hybrid are 7 days after inoculation $(\mathrm{DAI})=1.8,14 \mathrm{DAI}=3.5,21 \mathrm{DAI}=7.3$, and $28 \mathrm{DAI}=8.9$. 

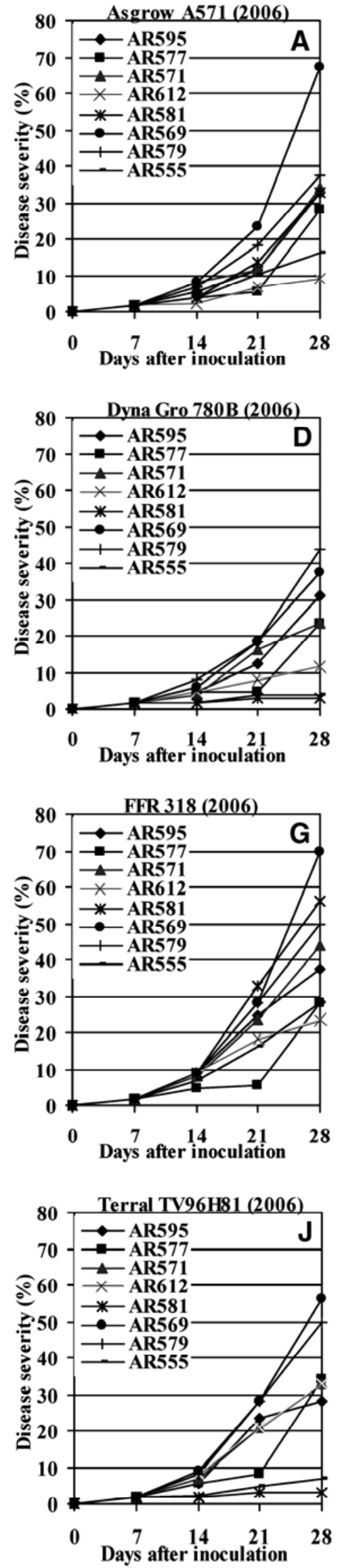
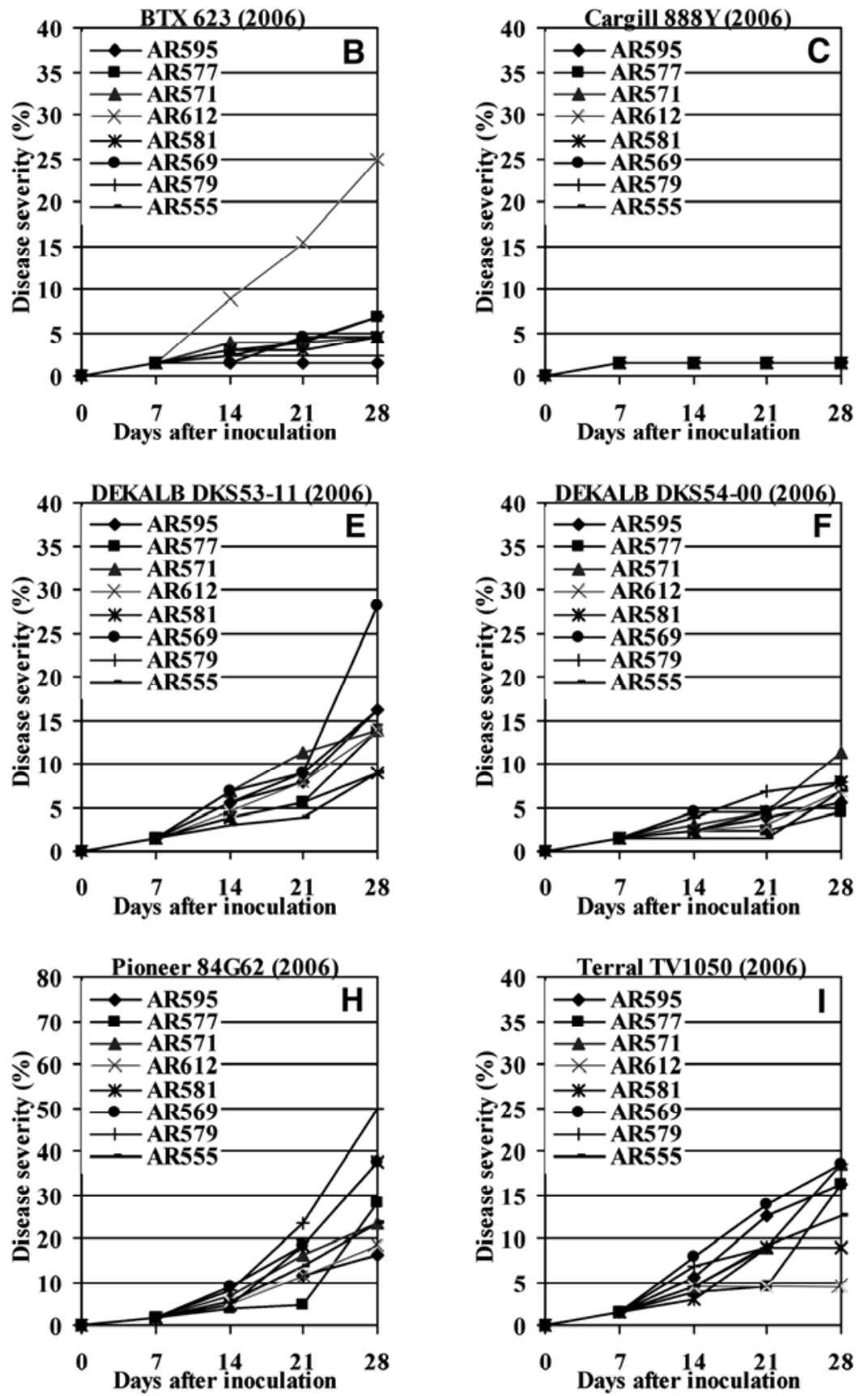

Fig. 2. Sorghum anthracnose development on 10 grain sorghum hybrids for eight Colletotrichum sublineolum isolates in the 2006 field test. Reported data represents the average of four replications. Least significant differences that compare isolates for the same or different grain sorghum hybrid are 7 days after inoculation $(\mathrm{DAI})=1.8,14 \mathrm{DAI}=3.5,21 \mathrm{DAI}=7.3$, and $28 \mathrm{DAI}=8.9$. 
reproduce on this sorghum hybrid (Figs. $1 \mathrm{C}$ and 2C).

Anthracnose severity ratings at 7 and 14 DAI were similar in 2005 and 2006 for Dyna Gro 780B (Figs. 1D and 2D). Isolates AR595, AR577, AR571, AR612, AR569, and AR579 produced approximately $20 \%$ disease by 28 DAI in 2005 . In 2006, each of these isolates, except for AR612, caused over 20\% disease on Dyna Gro 780B, with AR569 (pathotype 12) and AR579 (pathotype 13) producing the highest severity ratings (over $35 \%$ ) compared with all of the isolates tested. AR581 (pathotype 11) and AR555 (pathotype 14) had the lowest disease severity ratings of all the isolates and neither of these isolates produced severity ratings over approximately $5 \%$ in either year.

Severity ratings for DEKALB DKS5311 were similar in 2005 and 2006 for all isolates except for AR569 (pathotype 12) in 2006 (Figs. 1E and 2E). Disease ratings remained below $20 \%$ in 2005 for all isolates tested and, in 2006, for all isolates except AR569 that produced approximately $30 \%$ severity on DEKALB DKS5311. The severity of anthracnose progressed similarly in both years from the 7 DAI rating through the 28 DAI rating for six of the eight isolates evaluated. AR555 did not show an increase in severity from 7 to 28 DAI in 2005. However, in 2006, disease severity increased for this isolate from approximately $2 \%$ at 7 DAI to around $10 \%$ at 28 DAI. AR569 had produced similar levels of anthracnose on DEKALB DKS53-11 in 2005 and 2006 from the 7DAI rating through the 21-DAI rating but, in 2006, severity increased approximately $20 \%$ from the 21 - to the 28 -DAI rating and only increased $10 \%$ in 2005.

DEKALB DKS54-00 appeared to be more resistant to all the isolates than DEKALB DKS53-11. On average, severity ratings were at approximately $10 \%$ or below for all isolates tested in both years (Figs. 1F and 2F). However, AR571 and AR581 produced significantly more disease on this sorghum hybrid than the other six isolates in 2005. No significant differences were detected between isolates for severity ratings in 2006.

FFR 318 had one of the highest severity ratings of all 10 sorghum hybrids evaluated. In 2005, severity ratings at 28 DAI ranged from approximately 10 to $30 \%$ and, in 2006, ratings ranged from approximately 20 to $70 \%$ for all isolates (Figs. 1G and $2 \mathrm{G})$. None of the isolates resulted in ratings below $10 \%$ severity at $28 \mathrm{DAI}$ and only two isolates, AR555 in 2005 and AR577 in 2006, produced ratings below $10 \%$ at the 21-DAI rating. AR571, AR577, and AR581 produced the highest levels of disease in 2005, which was approximately $30 \%$. In contrast, AR569 produced the highest level of disease in $2006(70 \%)$ and AR581 caused approximately 55\% disease. However, two of the eight isolates produced similar amounts of disease in both years. FFR 318 inoculated with isolates AR577 (pathotype 8) and AR612 (pathotype 10) had severity ratings in 2005 and 2006 of approximately 30 and $20 \%$, respectively.

Disease severity averaged approximately $20 \%$ for six of the eight isolates at 28 DAI on Pioneer 84G62 in 2005 (Fig. $1 \mathrm{H})$. Isolate AR571 produced approximately $32 \%$ disease, which was significantly higher than any other isolate for this hybrid at 28 DAI in 2005 . In contrast, the 28-DAI severity rating for isolate AR555 was approximately 5\% and was significantly lower than any other isolate in 2005 . However, this was not the case in 2006. In 2006, severity ratings at 28 DAI ranged from approximately 15 to $50 \%$ for all isolates (Fig. 2H). AR579 caused the highest level of disease $(50 \%)$ at 28 DAI, with isolates AR581 and AR569 generating the next highest disease ratings, each at approximately $38 \%$. Isolate AR555 produced different levels of disease on Pioneer 84 G62 between years, with an average severity rating of 5\% in 2005 and $22 \%$ in 2006 at 28 DAI.

Severity ratings for Terral TV1050 were similar in 2005 and 2006 for all isolates at 28 DAI (Figs. 1I and 2I). Disease severity remained less than $20 \%$ in both years for all isolates. AR612 produced severity ratings of approximately $5 \%$ in both years, which was significantly lower than six of the eight isolates. AR555 did not show an increase in severity from 7 to 28 DAI in 2005 whereas, in 2006, disease severity increased for this isolate from approximately $2 \%$ at 7 DAI to approximately $12 \%$ at 28 DAI.

Terral TV96H81 had one of the highest severity ratings of all 10 sorghum hybrids evaluated. In 2005, severity ratings at 28 DAI ranged from approximately 5 to $40 \%$ whereas, in 2006, ratings ranged from approximately 2 to $58 \%$ for all isolates (Figs. 1J and 2J). However, two isolates (AR581 and AR555) produced ratings below $10 \%$ severity at 28 DAI in both years. AR569, AR571, and AR577 produced the highest levels of disease in 2005, which were more than $30 \%$ at 28 DAI. Isolate AR569 produced the most disease in 2006 (approximately 58\%) on Terral TV96H81. In addition, isolate AR579 produced an average severity rating of $50 \%$ in 2006 at 28 DAI, which was almost twice the disease severity this isolate produced in 2005.

\section{DISCUSSION}

The objective of this research was to assess the levels of resistance of selected grain sorghum hybrids grown in Arkansas to the recently identified $C$. sublineolum pathotypes found in Arkansas. The results of our experiments conducted over two different years demonstrated that most of the grain sorghum hybrids evaluated were susceptible to one or more of the $C$. sublineolum isolates recently identified in Arkansas. Only Cargill 888Y appeared to be resistant to all isolates evaluated in both years. Although BTX 623 has been used as a susceptible reference in previous reports $(8,20)$, it appeared to be moderately resistant or moderately susceptible to most of the isolates found in Arkansas, with the exception of isolate AR612 (pathotype 10) in both years and isolate AR581 (pathotype 11) in 2005. Isolate AR571 (pathotype 9) appeared to be the most aggressive pathotype to 7 of the 10 sorghum hybrids in 2005. However, in 2006, isolates AR569 and AR579 (pathotypes 12 and 13, respectively) caused more disease than AR571 to all sorghum hybrids except for DEKALB DKS54-00.

Significant differences were noted in the rate at which the disease progressed between years for some of the isolates, suggesting that there may have been environmental factors affecting the disease components of these isolates. $\mathrm{Li}$ and TeBeest (12) showed that one C. sublineolum strain can be detected at a higher frequency near the end of the growing season, although three strains were inoculated in equal proportions on the sorghum plants earlier in the growing season. A similar experiment showed that some isolates of C. gloeosporioides f. sp. aeschynomene can become the dominant strain within a population in the field even when other isolates of the same species are present in the field at the time of inoculation (7). Because sorghum anthracnose is a polycyclic disease, the time from infection to the production of new spores may have been increased in 2005 due to the small amount of rainfall in 2005 compared with the high rainfall amounts in 2006. However, isolate AR571 (pathotype 9) was not influenced by the different environmental conditions between years because disease severity was similar on each sorghum hybrid in both years. One reason for this difference may have been that the moisture requirements needed for spores to germinate and infect were met for AR571 even in 2005 when rainfall was minimal.

In contrast, several isolates caused more disease than isolate AR571 by 28 DAI in 2006, which was the wetter year of the 2year study. These results show the importance of evaluating resistance across years and in different environments. A similar result was observed in Mali, where 270 Malian sorghum accessions were evaluated for resistance to anthracnose during dry and wet seasons (8). That work showed a significant genotype-environment interaction and a higher percentage of the accessions were rated as more resistant in the dry season than in the wet season. Erpelding and Prom (8) also observed a delay in disease increase in the dry season when compared with the wet season. Their results may partially explain the environ- 
ment-isolate-sorghum hybrid interactions we observed in our experiments.

Overall, grain sorghum anthracnose can cause yield losses in susceptible hybrids grown in Arkansas (16). However, the amount of yield loss associated with anthracnose is not clear because some sorghum hybrids may appear to be susceptible but maintain low levels of disease while other susceptible hybrids may have higher amounts of anthracnose. However, our data show that resistance is available with the sorghum hybrid Cargill 888Y. This hybrid was resistant to isolate AR571 (pathotype 9), which has been found in Arkansas at the highest frequency and is virulent to all eight of the differential inbred breeding lines used to identify pathotypes $(4,15)$.

More work is needed to assess the interactions of host resistance, yield parameters, and pathotypes that infect the grain sorghum hybrids that are planted in Arkansas. In addition, more work is also needed to investigate the interactions of $C$. sublineolum on yield losses and effects on seed quality while grain is maturing on the plant, because $C$. sublineolum continues to colonize the entire sorghum plant even after the sorghum grain is ready for harvest.

\section{ACKNOWLEDGMENTS}

We thank the University of Arkansas, Division of Agriculture and the Arkansas Corn and Grain Sorghum Board for financial support of this research.

\section{LITERATURE CITED}

1. Ali, M. E. K., and Warren, H. L. 1987. Physiological races of Colletotrichum graminicola on sorghum. Plant Dis. 71:402-404.

2. Boora, K. S., Frederiksen, R., and Magill, C. 1998. DNA-based markers for a recessive gene conferring anthracnose resistance in sorghum.
Crop Sci. 38:1708-1709.

3. Campbell, C. L., and Madden, L. V. 1990. Introduction to Plant Disease Epidemiology. John Wiley and Sons, Inc., New York.

4. Cardwell, K. F., Hepperly, P. R., and Frederiksen, R. A. 1989. Pathotypes of Colletotrichum graminicola and seed transmission of sorghum anthracnose. Plant Dis. 73:255-257.

5. Casela, C. R., Frederiksen, R. A., and Ferreira, A. S. 1993. Evidence for dilatory resistance to anthracnose in sorghum. Plant Dis. 77:908911.

6. Dale, J. L., Kirkpatrick, T. L., and Cardwell, K. F. 1988. Sorghum anthracnose in southwest Arkansas. Ark. Farm Res. 37:17.

7. Ditmore, M., Moore, J. W., and TeBeest, D. O. 2008. Interactions of two selected field isolates of Colletotrichum gloeosporioides f. sp. aeschynomene on Aeschynomene virginica. Biol. Control 47:298-308.

8. Erpelding, J. E., and Prom, L. K. 2004. Evaluation of Malian sorghum germplasm for resistance against anthracnose. Plant Pathol. 3:65-71.

9. Frederiksen, R. A. 1986. Anthracnose stalk rot. Page 27 in: Compendium of Sorghum Diseases. Frederiksen, R. A. ed. American Phytopathological Society, St. Paul, MN.

10. Harris, H. B., Johnson, B. J., Dobson, J. W., and Luttrell, E. S. 1964. Evaluation of anthracnose on grain sorghum. Crop Sci. 4:460-462.

11. Li, Y. 2004. Temporal and spatial development, relative fitness and dynamics of strains of Colletotrichum sublineolum on grain sorghum. Doctoral dissertation, University of Arkansas, Fayetteville.

12. Li, Y. H., and TeBeest, D. O. 2009. Temporal and spatial development of sorghum anthracnose in Arkansas. Plant Dis. 93:287-292.

13. Little, T. M., and Hills, F. J. 1978. Agricultural Experimentation Design and Analysis. John Wiley and Sons, Inc., New York.

14. Moore, J. W. 2007. Characterizing pathotypes and haplotypes of Colletotrichum sublineolum in Arkansas. Doctoral dissertation, University of Arkansas, Fayetteville.

15. Moore, J. W., Ditmore, M., and TeBeest, D. O. 2008. Pathotypes of Colletotrichum sublineolum in Arkansas. Plant Dis. 92:1415-1420.

16. Moore, J. W., Ditmore, M., and TeBeest, D. O
2009. The effects of cropping history on grain sorghum yields and anthracnose severity in Arkansas. Crop Prot. 28:737-743.

17. Pande, S., Mughogho, L. K., Bandyopadhyay, R., and Karunakar, R. I. 1991. Variation in pathogenicity and cultural characteristics of sorghum isolates of Colletotrichum graminicola in India. Plant Dis. 75:778-783.

18. Rosewich, U. L., Pettway, R. E., McDonald, B. A., Duncan, R. R., and Frederiksen, R. A 1998. Genetic structure and temporal dynamics of a Colletotrichum graminicola population in a sorghum disease nursery. Phytopathology 88:1087-1093.

19. TeBeest, D., Kirkpatrick, T., and Cartwright, R. 2004. Common and important diseases of grain sorghum. In: Grain Sorghum Production Handbook. L. Espinoza and J. Kelley, eds. Univ. Ark. Coop. Ext. MP 297

20. Tenkouano, A., Miller, F. R., Hart, G. E., Frederiksen, R. A., and Nicholson, R. L. 1993. Phytoalexin assay in juvenile sorghum: an aid to breeding for anthracnose resistance. Crop Sci. 33:243-248.

21. Thakur, R. P., Mathur, K., Rao, V. P., Chandra, S., Shivramkrishnan, S., Kannan, S., Hiremath, R. V., Tailor, H. C., Kushwaha, U. S., Dwivedi, R. R., and Indira, S. 1998. Pathogenic and genetic characterization of six Indian populations of Colletotrichum sublineolum, the causal agent of sorghum anthracnose. Indian Phytopathol. 51:338-348.

22. Thomas, M. D., Sissoko, I., and Sacko, M. 1996. Development of leaf anthracnose and its effect on yield and grain weight of sorghum in West Africa. Plant Dis. 80:151-153.

23. Ware, S. B. 2001. Genetic diversity of Colletotrichum sublineolum based on vegetative compatibility, mtDNA and nuDNA restriction fragment length polymorphisms, and intron sequences. Masters thesis. University of Arkansas, Fayetteville.

24. Warren, H. L. 1986. Leaf Anthracnose. Pages 10-11 in: Compendium of Sorghum Diseases. R. A. Frederiksen, ed. American Phytopathological Society, St. Paul, MN

25. Warren, H. L. 1986. Panicle and Grain Anthracnose. Page 40 in: Compendium of Sorghum Diseases. R. A. Frederiksen, ed. American Phytopathological Society, St. Paul, MN 